\title{
Hepatitis $B$ and $C$ seroprevalence in patients with diabetes mellitus and its relationship with microvascular complications
}

\author{
Kadir Gisi ${ }^{1}$, Ali Cetinkaya ${ }^{1}$, Mesut Ozkaya², Bulent Kantarceken ${ }^{1}$, Gokce Gisi ${ }^{3}$, Sedat Koroglu ${ }^{4}$ \\ ${ }^{1}$ Gastroenterology Department, Faculty of Medicine, Sutcu Imam University, Kahramanmaras, Turkey \\ ${ }^{2}$ Endocrinology Department, Faculty of Medicine, Gaziantep University, Gaziantep, Turkey \\ ${ }^{3}$ Anesthesiology Department, Faculty of Medicine, Sutcu Imam University, Kahramanmaras, Turkey \\ ${ }^{4}$ Cardiology Clinic, Necip Fazil City Hospital, Kahramanmaras, Turkey
}

Gastroenterology Rev 2017; 12 (2): 105-110

DOI: https://doi.org/10.5114/pg.2016.64748

Key words: diabetes mellitus, hepatitis B prevalence, hepatitis C prevalence, microvascular complications.

Address for correspondence: Dr. Kadir Gisi, Gastroenterology Department, Sutcu Imam University, Avşar Kampüsü, Kahramanmaras,

Turkey, phone: +90 3442257575, e-mail: kadirgisi@gmail.com

\begin{abstract}
Introduction: Diabetic patients are susceptible to bacterial, viral and fungal infections because of various deficiencies in the immune system.

Aim: To investigate a possible link between hepatitis B/C prevalence and microvascular complications as well as duration of diabetes.

Material and methods: In total 1263 diabetic patients (1149 type 2, 114 type 1) were enrolled in the study. The control group consisted of 1482 healthy blood donors who were over 40 years old. All diabetic patients were tested for HBsAg, anti-HBs and anti-HCV beside routine laboratory tests. Diabetic patients were divided into three groups according to their diabetes duration, and all of the patients were scanned for microvascular complications. Demographic data of all patients were recorded.

Results: HBsAg seropositivity was $3.7 \%$ in diabetic patients and $1.08 \%$ in the control group; this difference was statistically significant $(p<0.001)$. HBsAg positivity rates in type 1 and type 2 diabetics were $0.8 \%$ and $4 \%$, respectively $(p=0.09)$. HCV seropositivity was $2.2 \%$ for diabetics and $0.5 \%$ for the control group; this difference was statistically significant $(p<0.001)$. Anti-HCV seropositivity in type 1 and type 2 diabetics was $1.75 \%$ and $2.26 \%$, respectively. There was no relationship between diabetes duration and hepatitis B-C prevalence $(p>0.05)$. Also, no relationship was found between microvascular complications of diabetes and hepatitis $B / C$ seropositivity.

Conclusions: Hepatitis B and C seroprevalence was found to be increased in diabetes mellitus; however, there was no relationship between hepatitis seroprevalence and the duration or microvascular complications of diabetes.
\end{abstract}

\section{Introduction}

Diabetes mellitus (DM), in which the organism cannot benefit from carbohydrates, fats, and proteins due to insulin deficiency or defects related to insulin and with which micro- and/or macrovascular complications may coexist over time, is a chronic metabolic disease that requires constant medical care $[1,2]$.

The relationship between diabetes and the liver has been known since 30 years ago. Although the main effect of diabetes on the liver is known as hepatic steatosis, diabetic patients are exposed to hepatitis virus (particularly hepatitis $C$ virus) infections, and for this reason it has been reported that the transaminase levels and the hepatitis serology of diabetic patients should be examined [3-6].

It is estimated that $350-400$ million people around the world are chronically infected with hepatitis $B$ virus (HBV) and 210 million people are chronically infected with hepatitis C virus (HCV) [7]. These two infectious agents are risk factors for the development of liver cirrhosis and liver carcinoma [8]. Hepatitis B and C can be found with many diseases epidemiologically. Also, their associations with diseases that suppress the immune system are frequent [9]. 
It has been reported that in many studies, hepatitis $B$ and hepatitis $C$ infections were detected more frequently in patients with DM. As HCV infection was put forward as a risk factor for DM, it was suggested that DM might also be a risk factor for HCV infection [9]. In the case-control study by Mason et al., the prevalence of HCV infection in individuals with diabetes was found to be significantly higher than the healthy controls (4.2\% vs. $1.6 \%)$ [10].

\section{Aim}

In this study, we aimed to investigate the frequency of hepatitis B and C by looking at HbsAg and anti-HCV serology in type I and type II diabetic patients and to show whether there is a relationship between microvascular complications of diabetes (neuropathy, retinopathy, and nephropathy), the duration of diabetes and the frequency of hepatitis B-C.

\section{Material and methods}

In total, 1263 type I and type II diabetics, who met the study criteria among 4700 DM patients who applied to Kahramanmaras Sutcu Imam University School of Medicine Hospital Gastroenterology, Endocrinology and General Internal Medicine outpatient clinics between November 2006 and January 2010, were included in the study. Our local ethics committee approved the study. Exclusion criteria were as follows: known autoimmune or immunosuppressive diseases, malignancy, patients who have received or are still receiving immunosuppressive drug therapy, patients who have had hepatitis $B$ vaccine, and patients with a history of hepatitis $B$ or $C$ before DM diagnosis. The control group was composed of 1482 otherwise healthy blood donors over the age of 40 who applied to the hospital between years 2006 and 2010.

The patients were divided into three groups according to the duration of diabetes: $0-5,6-10$ and $>10$ years. The following routine laboratory examinations of all patients were performed: blood count, fasting and postprandial blood glucose, full urinalysis, glycated hemoglobin $\left(\mathrm{HbA}_{1 \mathrm{c}}\right)$ level, blood urea nitro- gen (BUN), creatinine, aspartate aminotransferase (AST), alanine aminotransferase (ALT), and spot urine protein/creatinine. In addition to these examinations, $\mathrm{HbsAg}$, anti-HBs and anti-HCV examinations were requested from patients. The hepatitis serology was carried out with a Architect Reagent Kit, Wiesbaden, Germany device. In addition, the patients who applied to outpatient clinics were also screened in terms of microvascular complications of diabetes. The presence of diabetic nephropathy in patients was detected by looking at the full urinalysis, the blood creatinine level, and protein/creatinine ratio in spot urine. All of the patients with nephropathy were consulted with a nephrology clinic about the cause of nephropathy. Only the patients with confirmed diabetic nephropathy were included. The presence of neuropathy was detected by neurological examination of the patient, and the presence of retinopathy was detected by fundus examination by an eye disease specialist. Patients who had had a fundus examination within 6 months were not asked to have a fundus examination again.

\section{Statistical analysis}

All data were processed using the SPSS statistical software (version 18) and a $p$-value of less than 0.05 was considered to be significant. The Student $t$-test was used for the analysis of parametric measurements, and the $\chi^{2}$ test was used for non-parametric measurements.

\section{Results}

A total of 1263 diabetic patients and 1482 healthy blood donors as a control group were included in the study. In the diabetic group, 114 patients had type I and the remaining 1149 patients had type II diabetes; their average age was $24.54 \pm 10.59$ and $56.11 \pm 12.71$, respectively. The demographic characteristics of patients are shown in Table I.

The number of $\mathrm{HbsAg}$ positive patients among diabetics was 47 (3.7\%). Out of these, 1 person was type I (1.08\%) and 46 (4\%) people were type II diabetic. There was no significant difference in gender and type of diabetes in terms of $\mathrm{HbsAg}$ positivity

Table I. The demographic characteristic of the patients and controls

\begin{tabular}{|c|c|c|c|c|c|c|}
\hline \multirow[t]{2}{*}{ Study groups } & \multicolumn{3}{|c|}{ HBsAg (+) } & \multicolumn{3}{|c|}{ Anti-HCV (+) } \\
\hline & Diabetic & Control & $P$-value & Diabetic & Control & $P$-value \\
\hline Number, n (\%) & $47(3.7)$ & $16(1.08)$ & $<0.001$ & $28(2.2)$ & $7(0.5)$ & $<0.001$ \\
\hline Age & $55.8 \pm 15.4$ & $46.93 \pm 5.8$ & $<0.001$ & $57.96 \pm 16.24$ & $47.14 \pm 13.56$ & $<0.001$ \\
\hline Male, $n(\%)$ & $20 / 472(4.2)$ & $16 / 1464$ (1.09) & $>0.05$ & $8 / 472$ (1.7) & $7 / 1464(0.5)$ & $>0.05$ \\
\hline Female, $n(\%)$ & 27/791 (3.4) & 0/18 (0) & $>0.05$ & 20/791 (2.5) & 0/18 (0) & $>0.05$ \\
\hline
\end{tabular}


$(p>0.05)$. The number of anti-HCV positive patients was $28(2.2 \%)$. Two (1.75\%) of them were type I diabetic and 26 (2.26\%) were type II diabetic. There was no significant difference in gender, type of diabetes or anti-HCV positivity $(p>0.05)$. In addition, 9 of these anti-HCV positive patients were found to be immune to hepatitis $B$. There was no patient infected with both hepatitis $B$ and $C$.

One thousand and four hundred eighty-two donors over the age of 40 were included in the study. The reason for including ages over 40 was to provide an average age close to the study patients. The average age of the donors was $47.53 \pm 5.8$. There was a significant difference between the patient group and donors in terms of age and gender $(p<0.001)$. When these two groups were compared in terms of hepatitis B and C, again the difference between two groups was significant $(p<0.001)$.
The patients were divided into 3 groups according to the duration of diabetes. It was found that in three groups, there was no significant increase in the frequency of hepatitis B and C with the increase in duration of diabetes $(p>0.05)$. However, although it was not statistically significant, with the increase of the duration of diabetes, HCV frequency increased, especially in type I diabetes (see Table II).

Also, the relationship between diabetes complications and hepatitis was analyzed. As a result, no statistical relationship was found between neuropathy, retinopathy and nephropathy with HBV and anti-HCV positivity (Table III).

When hepatitis B and C positive patients were compared with each other in terms of laboratory results, there was no significant difference between the 2 patient groups except postprandial blood glucose (PBG) $(p>0.05)$. PBG was higher in patients with HBV. The

Table II. Relationship between diabetes duration and HBV and HCV seroprevalence

\begin{tabular}{|c|c|c|c|c|c|c|}
\hline \multirow{2}{*}{$\begin{array}{l}\text { Duration of DM } \\
\text { [years] }\end{array}$} & \multicolumn{3}{|c|}{ HBV seroprevalence } & \multicolumn{3}{|c|}{ HCV seroprevalence } \\
\hline & $\begin{array}{c}\text { HBsAg (+) } \\
n(\%)\end{array}$ & $\begin{array}{c}\text { HBsAg (-) } \\
n(\%)\end{array}$ & $P$-value & $\begin{array}{c}\text { Anti-HCV (+) } \\
n(\%)\end{array}$ & $\begin{array}{c}\text { Anti-HCV (-) } \\
n(\%)\end{array}$ & $P$-value \\
\hline \multicolumn{7}{|l|}{$0-5:$} \\
\hline Type I & $0(0)$ & $56(49.1)$ & 0.185 & $0(0)$ & $56(49.1)$ & 0.290 \\
\hline Type II & $14(1.2)$ & $444(38.6)$ & & $9(0.8)$ & 449 (39.1) & \\
\hline \multicolumn{7}{|l|}{ 6-10: } \\
\hline Type I & $1(0.9)$ & $28(24.6)$ & 0.770 & $0(0)$ & $29(2.5)$ & 0.500 \\
\hline Type II & $15(1.3)$ & 309 (26.9) & & $5(0.4)$ & $319(27.8)$ & \\
\hline \multicolumn{7}{|l|}{$>10$} \\
\hline Type I & $0(0)$ & $29(25.4)$ & 0.236 & $2(1.8)$ & $27(23.7)$ & 0.309 \\
\hline Type II & $17(1.5)$ & $350(30.5)$ & & $12(1.0)$ & 355 (30.9) & \\
\hline
\end{tabular}

Table III. Relationship between diabetic microvascular complications and hepatitis seroprevalence

\begin{tabular}{|c|c|c|c|c|}
\hline Complication & Hepatitis (-), $n(\%)$ & HBV (+), $n(\%)$ & $\mathrm{HCV}(+), n(\%)$ & $P$-value \\
\hline \multicolumn{5}{|l|}{ Neuropathy: } \\
\hline Positive & $740(62.3)$ & $35(74.4)$ & $19(67.8)$ & 0.204 \\
\hline Negative & $448(47.7)$ & $12(25.6)$ & $9(32.2)$ & \\
\hline \multicolumn{5}{|l|}{ Retinopathy: } \\
\hline Positive & $446(37.5)$ & $19(40.4)$ & $14(50)$ & 0.380 \\
\hline Negative & $742(62.5)$ & $28(59.6)$ & $14(50)$ & \\
\hline \multicolumn{5}{|l|}{ Nephropathy: } \\
\hline Positive & $291(24.5)$ & $16(34)$ & $10(35.7)$ & 0.142 \\
\hline Negative & 897 (75.5) & $31(66)$ & $18(64.3)$ & \\
\hline
\end{tabular}


level of liver enzyme among hepatitis $B$ and $C$ positive patients was found to be high in 7 of 47 patients with HBV (14.9\%) and was found to be high in 4 of 28 patients with HCV (14.3\%).

\section{Discussion}

The relationship between diabetes and the liver has been known for more than 30 years. In the studies, the movements of polymorphonuclear leukocytes (PNL) in diabetic patients towards the area of inflammation were shown to be slower than those of healthy subjects [11-13]. For this reason and because of many reasons that cannot be explained fully, diabetic patients become susceptible to various bacterial, viral and fungal infections. Mucormycosis, malignant external otitis, emphysematous pyelonephritis and emphysematous cholecystitis diseases are known to be strongly associated with diabetes [11]. On the other hand, in the studies on hepatitis immunology in patients with DM, it was found that diabetic patients generated a significantly lower antibody response compared to healthy subjects [14-16]. While the frequency of HCV infection worldwide is around $3 \%$, the frequency of HBV infection varies between $0.1 \%$ and $20 \%$. In the community-wide screening in our country, the HBV infection frequency varies in the range $1.7-21 \%$, and HCV frequency varies in the range $1-2.4 \%[7,17]$.

Although the relationship between HCV infection and DM still cannot be explained fully, some hypotheses have been proposed claiming that DM may be an extrahepatic manifestation of HCV infection and DM emerges through metabolic changes that are generated by HCV's hepatosteatosis [18-20]. In most of the studies on the prevalence of HCV infection, it has been shown that HCV prevalence was higher in diabetic patients compared to non-diabetic patients [21-24]. Different results were obtained from studies conducted on this topic. In a study conducted in Spain on 176 diabetics and 6172 blood donors, it was found that the $\mathrm{HCV}$ prevalence in the diabetic group was significantly higher than the donor group (11\% and $2.5 \%$ respectively, $p<0.001$ ) [25]. In the study conducted by Mason et al., a higher HCV rate was detected in diabetic patients as $4.2 \%$ vs. $1.6 \%$ [10]. In a large-scale study on 820 diabetics and 905 healthy controls, it was found that HCV prevalence increased 2.8 times more than the control group $(p<0.001)$ and type II DM patients had 2.8 times increased risk of HCV [22]. In some of the studies conducted in our country, HCV prevalence in diabetic patients has been found to be higher than the control groups $[23,26]$. In contrast to these studies, Sotiropoulos et al. reported that HCV prevalence in diabetic patients in Greece was low (1.65\%) compared to the normal society average (2\%) [27]. On the other hand, in our study, HCV seropositivity in diabetic patients was found to be $2.2 \%$ and HCV seropositivity in the control group was found to be $0.5 \%$. The difference between the two groups was statistically significant $(p<0.001)$. However, this rate was similar to the average of the general population. We also looked at the seroprevalence separately in type I and type II diabetes and found it to be $1.75 \%$ in type I and as $2.26 \%$ in type II DM. The difference between type I and type II DM was not statistically significant. Only a few studies have assessed the HCV serology between types of diabetes. Among these studies, in the study by Gülcan et al., no difference was detected between type I and type II DM in terms of HCV seropositivity [26].

The prevalence of HBV infection in diabetic patients has been investigated in very few studies. In the study by Sangiorgio et al., the HBV prevalence in diabetic patients was found to be significantly higher than in the control group (7.1\% vs. $1.6 \% ; p<0.001)$ [28]. In contrast, in other studies HBV seroprevalence in diabetic patients was not different from the control groups [22, $23,26,29]$. In our study, HbsAg seropositivity in diabetic patients was $3.7 \%$ and $1.08 \%$ in the control group. The difference between the control group and diabetic groups was statistically significant $(p<0.001)$. Additionally, $\mathrm{HbsAg}$ positivity rates in type I and type II diabetics were $0.8 \%$ and $4 \%$ respectively. The difference between diabetes subgroups was not statistically significant $(p=0.09)$. This was ascribed to the small number of type I DM patients.

In addition, in some studies, the relationship between the duration of diabetes and the hepatitis serology in diabetic patients has been investigated [23, 26]. In these studies, different results were obtained. Okan et al. found that the anti-HCV positivity in patients having diabetes duration over 12 years was statistically significant compared to patients having lower diabetes duration $(p<0.05)$ [23]. However, they found no significant difference in terms of hepatitis B. Gülcan et al. found $\mathrm{HbsAg}$ positivity as significantly high in patients with diabetes over 10 years [26]. In contrast to these studies, Ali et al. found in their studies that anti-HCV positivity in patients with duration of diabetes lower than 5 years was significantly higher, and they stated that the duration of diabetes did not pose a risk for HCV infection [30]. Sangiorgio et al. failed to find a significant relationship between either type of hepatitis and the duration of diabetes [28]. We also found no significant relationship between the duration of diabetes and hepatitis $B$ and $C$ positivity.

Also, in our study, we examined the relationship between chronic complications of diabetes and $\mathrm{HbsAg}$ 
and anti-HCV positivity, which has been analyzed only in one study so far. In the study by Gülcan et al., only chronic complications were mentioned, and its relationship between hepatitis $B$ and $C$ positivity has not been evaluated [26]. In our study, we also examined the relationship between neuropathy, retinopathy, and nephropathy, which are chronic microvascular complications of diabetes, and HBV and HCV positivity. We found no significant relationship between microvascular complications and HBV and HCV positivity.

In most of the studies around the world, blood donors were used as the control group. We also chose healthy blood donors in our study. People who know that they are HBV or HCV positive will not come to give blood, and therefore it could be thought that this may not reflect the normal society average. This aspect may be a limitation of our study. On the other hand, another limitation of our study is that we did not question the patients' operation history, previous blood transfusion history, the presence of hepatitis-positive members in the family, and the history of drug use, because in previous studies, hospitalization, insulin usage, and blood transfusion have been suggested as risk factors for the development of hepatitis.

\section{Conclusions}

In our study, we compared diabetic patients with healthy blood donors over the age of 40 . We found that HBV and HCV frequency in diabetic patients was higher than donors, and our results were statistically significant. Also, we found no significant relationship between the duration of diabetes and hepatitis positivity (except anti-HCV positivity in type I DM). We concluded that there is no significant difference between type I and type II DM in terms of hepatitis positivity and also that there is no relationship between hepatitis positivity and age and gender. As a result, it can be argued that DM may increase the frequency of HBV and HCV infections. In addition, this study showed that the frequency of hepatitis $B$ and $C$ incidence in diabetic patients does not change with the frequency of microvascular complications (neuropathy, retinopathy, and nephropathy). We believe that multicenter studies that cover larger societies are needed in order to obtain greater understanding of this issue.

\section{Conflict of interest}

The authors declare no conflict of interest.

\section{References}

1. The Society of Endocrinology and Metabolism of Turkey. The guideline of diabetes mellitus and its complications 2009; 15-28.
2. American Diabetes Association. Diagnosis and classification of diabetes mellitus. Diabetes Care 2004; 27: 5-14.

3. Clark JM, Brancati FL, Diehl AM. The prevalence and etiology of elevated aminotransferase levels in the United States. Am J Gastroenterol 2003; 98: 960-7.

4. Jin HB, Gu ZY, Yu CH, et al. Association of nonalcoholic fatty liver disease with type 2 diabetes: clinical features and independent risk factors in diabetic fatty liver patients. Hepatobiliary Pancreat Dis Int 2005; 4: 389-92.

5. Gray H, Wreghitt T, Stratton IM, et al. High prevalence of hepatitis $\mathrm{C}$ infection in Afro-Caribbean patients with type $2 \mathrm{di}$ abetes and abnormal liver function tests. Diabet Med 1995; 12: 244-9.

6. Gupte P, Amarapurkar D, Agal S, et al. Non-alcoholic steatohepatitis in type 2 diabetes mellitus. J Gastroenterol Hepatol 2004; 19: 854-8.

7. Sumbul M. Epidemiology and protection of HCV infection. In: Viral Hepatitis. Tabak F, Balık I, Tekeli E (eds). Oban Press 2007; 208-19.

8. Wang J, Zhao H, Zhao S. Prevalence of HCV and HBV infection in patients with primary hepatocellular carcinoma in Shanxi Province. Zhonghua Liu Xing Bing Xue Za Zhi 1999; 20: 215-7.

9. Robinson WS. Biology of human hepatitis viruses. In: Hepatology: A Textbook of Liver Disease. Zakim D, Boyer TD (eds). WB Saunders Company 1996; 1146-91.

10. Mason AL, Lau JY, Hoang N, et al. Association of diabetes mellitus and chronic hepatitis C virus infection. Hepatology 1999; 29: 328-33.

11. Sentochnik DE, Eliopoulos G. Infection and diabetes. In: Joslin's Diabetes Mellitus. Kahn CR, Weir GC (eds). Lippincott Williams \& Wilkins 2005; 1018-33.

12. Molenaar DM, Palumbo PJ, Wilson WR, et al. Leukocyte chemotaxis in diabetic patients and their nondiabetic first-degree relatives. Diabetes 1976; 25: 880-3.

13. Mowat A, Baum J. Chemotaxis of polymorphonuclear leukocytes from patients with diabetes mellitus. N Engl J Med 1971; 284: 621-7.

14. Fiçicioğlu C, Mikla S, Midilli K, et al. Reduced immune response to hepatitis $B$ vaccine in children with insulin dependent diabetes. Acta Paediatr Jpn 1995; 37: 687-90.

15. Douvin C, Simon D, Charles MA, et al. Hepatitis B vaccination in diabetic patients. Randomized trial comparing recombinant vaccines containing and not containing pre-S2 antigen. Diabetes Care 1997; 20: 148-51.

16. Wismans PJ, van Hattum J, de Gast GC, et al. A prospective study of in vitro anti-HBs producing B cells (spot-ELISA) following primary and supplementary vaccination with a recombinant hepatitis B vaccine in insulin dependent diabetic patients and matched controls. J Med Virol 1991; 35: 216-22.

17. Ozdemir D, Kurt H. Epidemiology of Hepatitis B infections. In: Viral Hepatitis. Tabak F, Balik I, Tekeli E (eds). Viral Hepatitis Society Press 2007; 108-16.

18. Allison ME, Wreghitt T, Palmer CR, et al. Evidence for a link between hepatitis $C$ virus infection and diabetes mellitus in a cirrhotic population. J Hepatol 1994; 21: 1135-9.

19. Alexander GJ. An association between hepatitis $C$ virus infection and type 2 diabetes mellitus: what is the connection? Ann Intern Med 2000; 133: 650-2. 
20. Zein NN. Hepatitis C and diabetes mellitus: an ongoing controversy. Am J Gastroenterol 1998; 93: 2320-2.

21. Gurbuz AK, Ozel M, Demirturk L, et al. Frequency of anti HCV seropositivity in patients with type II diabetes mellitus. T Clin J Gastroenterohepatol 1999; 10: 113-6.

22. Chen HF, Li CY, Chen P, et al. Seroprevalence of hepatitis B and C in type 2 diabetic patients. J Chin Med Assoc 2006; 69: 146-52.

23. Okan V, Araz M, Aktaran S, et al. Increased frequency of HCV but not HBV infection in type 2 diabetic patients in Turkey. Int J Clin Pract 2002; 56: 175-7.

24. Parolin MB, Réa R, Vargas RM, et al. Prevalence of hepatitis C infection in patients with type 2 diabetes mellitus. Arq Gastroenterol 2006; 43: 77-80.

25. Simó R, Hernández C, Genescà J, et al. High prevalence of hepatitis C virus infection in diabetic patients. Diabetes Care 1996; 19: 998-1000.

26. Gülcan A, Gulcan E, Toker A, et al. Evaluation of risk factors and seroprevalence of hepatitis $B$ and $C$ in diabetic patients in Kutahya, Turkey. J Investig Med 2008; 56: 858-63.

27. Sotiropoulos A, Peppas TA, Skliros E, et al. Low prevalence of hepatitis $C$ virus infection in Greek diabetic patients. Diabet Med 1999; 16: 250-2.

28. Sangiorgio L, Attardo T, Gangemi R, et al. Increased frequency of HCV and HBV infection in type 2 diabetic patients. Diabetes Res Clin Pract 2000; 48: 147-51.

29. Savagnone E, Caruso V, Mondello P, et al. Hepatitis B virus in diabetic patients. Acta Diabetol Lat 1980; 17: 207-11.

30. Ali SS, Ali IS, Aamir AH, et al. Frequency of hepatitis C infection in diabetic patients. J Ayub Med Coll Abbottabad 2007; 19: 46-9.

Received: 28.08 .2015

Accepted: 12.01 .2016 